\title{
RESIST-PC: U.S. Academic Foray into PSMA Theranostic Trials
}

\author{
Amir Iravani ${ }^{1}$ and Thomas A. Hope ${ }^{2}$ \\ ${ }^{1}$ Mallinckrodt Institute of Radiology, Washington University in St. Louis, St. Louis, Missouri; and ${ }^{2}$ Department of Radiology and \\ Biomedical Imaging, University of California San Francisco, San Francisco, California
}

\section{See the associated article on page 1440.}

$\mathbf{I}_{\mathrm{n}}$ this issue of The Journal of Nuclear Medicine, Calais et al. from UCLA present the results of their phase 2 RESIST-PC trial (1). This trial predated the VISION trial and enrolled patients prospectively in a 2-arm study intended to compare the efficacy and safety of ${ }^{177} \mathrm{Lu}-\mathrm{PSMA}-617$ dosed at either 6.0 or $7.4 \mathrm{GBq}$ (2). The study was performed collaboratively between UCLA and Excel Diagnostics, although only the 43 patients enrolled at UCLA are presented in the article. The UCLA team must be commended for the effort in initiating and performing this study without company support. The effort required to open the first ${ }^{177}$ Lu-PSMA trial in the United States cannot be understated.

The absence of support for this study required that a cost recovery mechanism be used, something that is not commonly leveraged for therapeutic trials. Unlike in the Australian phase 2 study (3), in which the ${ }^{177} \mathrm{Lu}$ was provided free of charge from the Australian Nuclear Science and Technology Organization, the study team had to procure ${ }^{177} \mathrm{Lu}$ at cost. Prior diagnostic costrecovery trials led to the approval of both ${ }^{68} \mathrm{Ga}$-DOTATOC and ${ }^{68}$ Ga-PSMA-11 $(4,5)$. It may seem odd that there was no corporate support for this study given the large interest in the field we see today, but at the time of trial design, there was limited corporate interest. Similar to cost recovery, many European studies have leveraged compassionate use in the absence of company support (6).

Looking at the results presented for the RESIST-PC trial, the prostate-specific antigen (PSA) response ( $\geq 50 \%$ PSA decline) was $37 \%$, which is lower than reported in the LuPSMA (64\%) and TheraP $(66 \%)$ trials $(1,3,7)$. Although the inclusion criterion for prostate-specific antigen (PSMA) expression was not predefined in the RESIST-PC trial, the difference in PSA response may be accounted for by a lower threshold of PSMA PET avidity. The LuPSMA trial required an $\mathrm{SUV}_{\max }$ that was one and a half times that of the liver, whereas the TheraP trial required an $\mathrm{SUV}_{\max }$ of 20 at one site and no measurable disease below an $\mathrm{SUV}_{\max }$ of 10. In addition, the LuPSMA and TheraP trials used ${ }^{18} \mathrm{~F}$-FDG PET/CT to exclude patients with disease heterogeneity and sites of disease

Received June 3, 2021; revision accepted July 21, 2021.

For correspondence or reprints, contact Thomas A. Hope (thomas.hope@ ucsf.edu).

COPYRIGHT (C) 2021 by the Society of Nuclear Medicine and Molecular Imaging. demonstrating low PSMA expression. In the LuPSMA and TheraP trials, $25 \%-30 \%$ of patients were excluded, whereas in the RESIST-PC trial only 2 patients $(<5 \%)$ were excluded on the basis of PSMA expression. PSMA expression is critical, as shown by Violet et al., who demonstrated a positive correlation between pretreatment PSMA uptake and posttreatment dosimetry on a whole-body scale, and as further supported by Seifert et al., who showed that low average PSMA expression is a negative prognostic factor $(8,9)$.

The VISION trial used a lower cutoff of a PSMA-positive lesion greater than liver uptake with no negative PSMA lesions, which resulted in $13 \%$ of patients being excluded, more than twice as many as in the RESIST-PC study (2). In the VISION trial, $46 \%$ of patients treated with ${ }^{177}$ Lu-PSMA-617 had a greater than $50 \%$ reduction, a PSA response rate between the RESIST-PC trial and the TheraP/LuPSMA studies, again supporting the idea that the higher the cutoff for PSMA positivity combined with ${ }^{18} \mathrm{~F}-\mathrm{FDG}$ imaging, the better the response to treatment (2).

Although it appears that the higher threshold for PSMA avidity would result in a higher response rate, the threshold of PSMA avidity below which the patients may not respond to treatment remains unclear. It is also possible that patients with a limited volume of the discordant ${ }^{18} \mathrm{~F}-\mathrm{FDG}$-avid disease may derive some benefit from ${ }^{177}$ Lu-PSMA, subject to sufficient PSMA expression at other sites and as long as a more intensive therapeutic strategy is adopted. This possibility may support the combination with other oncologic treatments to tackle sites that may have been suboptimally targeted by ${ }^{177}$ Lu-PSMA. Multiple phase I/II combination regimens are under way using immunotherapy (NCT03658447, NCT03805594), poly(adenosine diphosphateribose) polymerase inhibitor (NCT03874884), androgen receptor-targeted therapy (NCT04419402), and even tandem treatment with chemotherapy in the castration-sensitive state (NCT03828838).

A separate issue with ${ }^{177}$ Lu-PSMA therapy is that the optimal treatment schedule is not well understood, including the administered activity per cycle, the interval between treatments, and the number of treatments/cumulative activity (10). The choice of a fixed administered activity of between 6 and $8 \mathrm{GBq}$ and up to 6 cycles is based predominantly on the limits of normal-organ absorbed dose and thresholds extrapolated from external-beam radiotherapy, ignoring fundamental differences in the radiobiology of radiopharmaceutical therapies. One of the most interesting aspects of RESIST-PC was that it attempted to determine the difference in efficacy and toxicity between 2 different doses of 
${ }^{177}$ Lu-PSMA-617, although the narrow difference between the doses and the premature closure of the study prevented the team from determining which dose was superior. Determination of the appropriate number of cycles, dose per cycle, and timing between cycles still remains an art form in radioligand therapy, although it is under study; for example, Weill Cornell is studying 2 higher dose cycles (up to $11.1 \mathrm{GBq}$ ) given 2 wk apart (NCT03042468).

It seems self-evident that straying from the rigid treatment plans used in these trials would be beneficial. Examples to consider include continuing therapy beyond 6 cycles in a subset of patients that continues to benefit from treatment, increasing intervals beyond $6-8 \mathrm{wk}$ in early responders, or rechallenging treatment at the time of progression, subject to sufficient target expression (11). Furthermore, incorporating posttreatment dosimetry will enhance our understanding of differences in absorbed doses in tumor and critical organs and how they impact patient outcome. Although the oversimplified approach of one size fits all would expedite the approval and increase the accessibility of this treatment, these advantages should not hinder exploiting the fundamental strengths of this treatment modality that allows individualizing the treatment on the basis of the patient's characteristics and tumor biology, as well as dynamically modifying the treatment schedule on the basis of response and posttreatment dosimetry.

It is unfortunate that completion of the RESIST-PC study was halted when the VISION trial started enrollment, as evaluating the difference between 2 different doses would have provided valuable information to the community. As we patiently await the approval of ${ }^{177} \mathrm{Lu}-\mathrm{PSMA}-617$ in light of the positive overall survival data from the VISION study, we would like to encourage members of the nuclear medicine community to develop and engage in multiinstitutional trials and to participate in National Cancer Institute cooperative groups, similar to what has proven successful in Australia.

\section{DISCLOSURE}

Thomas A. Hope is a consultant for Curium, and he received fees from Blue Earth Diagnostics and Ipsen outside the submitted work. No other potential conflict of interest relevant to this article was reported.

\section{REFERENCES}

1. Calais J, Gafita A, Eiber MR, et al. Prospective phase 2 trial of PSMA-targeted molecular radiotherapy with ${ }^{177}$ Lu-PSMA-617 for metastatic castration-resistant prostate cancer (RESIST-PC): efficacy results of the UCLA cohort. J Nucl Med. 2021;62:1440-1446.

2. Sartor O, de Bono J, Chi KN, et al. Lutetium-177-PSMA-617 for metastatic castration-resistant prostate cancer. $N$ Engl J Med. June 23, 2021 [Epub ahead of print].

3. Hofman MS, Sandhu S, Eu P, et al. Lutetium-177 PSMA (LuPSMA) theranostics phase II trial: efficacy, safety and QoL in patients with castrate-resistant prostate cancer treated with LuPSMA. Ann Oncol. 2017;28(supp1 5):v270.

4. Sartor O, Hope TA, Calais J, Fendler WP. Oliver Sartor talks with Thomas A. Hope, Jeremie Calais, and Wolfgang P. Fendler about FDA approval of PSMA. J Nucl Med. 2021;62:146-148.

5. Sunderland JJ. The academic NDA: justification, process, and lessons learned. J Nucl Med. 2020;61:480-487.

6. Rahbar K, Ahmadzadehfar H, Kratochwil C, et al. German multicenter study investigating ${ }^{177} \mathrm{Lu}$-PSMA-617 radioligand therapy in advanced prostate cancer patients. J Nucl Med. 2017;58:85-90.

7. Hofman MS, Goh JC, Tan TH, et al. $\left[{ }^{177} \mathrm{Lu}\right] \mathrm{Lu}-\mathrm{PSMA}-617$ versus cabazitaxel in patients with metastatic castration-resistant prostate cancer (TheraP): a randomised, open-label, phase 2 trial. Lancet. 2021;397:797-804.

8. Seifert R, Seitzer K, Herrmann K, et al. Analysis of PSMA expression and outcome in patients with advanced prostate cancer receiving ${ }^{177} \mathrm{Lu}$-PSMA-617 radioligand therapy. Theranostics. 2020;10:7812-7820.

9. Violet J, Jackson P, Ferdinandus J, et al. Dosimetry of ${ }^{177}$ Lu-PSMA-617 in metastatic castration-resistant prostate cancer: correlations between pretherapeutic imaging and whole-body tumor dosimetry with treatment outcomes. J Nucl Med. 2019;60:517-523.

10. Iravani A, Violet J, Azad A, Hofman MS. Lutetium-177 prostate-specific membrane antigen (PSMA) theranostics: practical nuances and intricacies. Prostate Cancer Prostatic Dis. 2020;23:38-52.

11. Violet J, Sandhu S, Iravani A, et al. Long-term follow-up and outcomes of retreatment in an expanded 50-patient single-center phase II prospective trial of $177 \mathrm{Lu}-$ PSMA-617 theranostics in metastatic castration-resistant prostate cancer. $J$ Nucl Med. 2020;61:857-865. 